\title{
Water fluxes through aquaporin-9 prime epithelial cells for rapid wound healing
}

\author{
Thommie Karlsson, Christoffer B. Lagerholm, Elena Vikström, \\ Vesa Loitto and Karl-Eric Magnusson
}

\section{Linköping University Post Print}

N.B.: When citing this work, cite the original article.

Original Publication:

Thommie Karlsson, Christoffer B. Lagerholm, Elena Vikström, Vesa Loitto and Karl-Eric Magnusson, Water fluxes through aquaporin-9 prime epithelial cells for rapid wound healing, 2013, Biochemical and Biophysical Research Communications - BBRC, (430), 3, 993-998.

http://dx.doi.org/10.1016/j.bbr.c.2012.11.125

Copyright: Elsevier http://www.elsevier.com/

Postprint available at: Linköping University Electronic Press

http://urn.kb.se/resolve?urn=urn:nbn:se:liu:diva-89749 


\section{Water Fluxes Through Aquaporin-9 Prime Epithelial Cells for Rapid}

\section{Wound Healing}

Thommie Karlsson $_{1}$, B. Christoffer Lagerholm 2 , Elena Vikström 1 , Vesa M. Loitto 1 and KarlEric Magnusson $_{1}$

${ }_{1}$ Division of Medical Microbiology, Department of Clinical and Experimental Medicine, Faculty of Health Sciences, Linköping University, SE-58185 Linköping, Sweden

${ }_{2}$ Department of Physics, Chemistry and Pharmacy, MEMPHYS - Center for Biomembrane Physics \& Danish Molecular Biomedical Imaging Center, University of Southern Denmark, CampusVej 55, DK-5230 Odense,

Denmark

E-mail addresses:

Thommie Karlsson_thommie.karlsson@liu.se

B. Christoffer Lagerholm lagerholm@sdu.dk

Elena Vikström elena.vikstrom@liu.se

Vesa M. Loitto_ vesa.loitto@liu.se

Karl-Eric Magnusson_karl-eric.magnusson@liu.se

\section{Correspondence to:}

Thommie Karlsson

E-mail: thommie.karlsson@liu.se

Address: Division of Medical Microbiology, Department of Clinical and Experimental Medicine, Faculty of Health Sciences, Linköping University, SE-58185, Linköping, Sweden

Telephone: $\quad+46(0) 707595925$

Fax: $\quad+46(0) 13224789$ 


\begin{abstract}
Cells move along surfaces both as single cells and multi-cellular units. Recent research points toward pivotal roles for water flux through aquaporins (AQPs) in single cell migration. Their expression is known to facilitate this process by promoting rapid shape changes. However, little is known about the impact on migrating epithelial sheets during wound healing and epithelial renewal. Here, we investigate and compare the effects of AQP9 on single cell and epithelial sheet migration. To achieve this, MDCK-1 cells stably expressing AQP9 were subjected to migration assessment. We found that AQP9 facilitated cell locomotion at both the single and multi-cellular level. Furthermore, we identified major differences in the monolayer integrity and cell size upon expression of AQP9 during epithelial sheet migration, indicating a rapid volume-regulatory mechanism. We suggest a novel mechanism for epithelial wound healing based on AQP-induced swelling and expansion of the monolayer.
\end{abstract}

Keywords: cell migration; aquaporins; AQP9; wound healing; cell motility

\author{
Abbreviation: \\ $\mathrm{AQP}$ - Aquaporin \\ CMV - Cytomegalovirus \\ PFA - Paraformaldehyde \\ NA - Numerical Aperture \\ GFP - Green fluorescent protein \\ EGF - Epidermal growth factor
}




\section{INTRODUCTION}

Cell migration is evident both for single cells and multi-cellular units. Despite distinct differences in the migration patterns, both these modes depend on a highly dynamic membrane, major cytoskeletal remodeling and cell-environmental interactions. Epithelial sheet migration is a common form of multi-cellular motility and is physiologically relevant at the skin and intestine during wound healing $[1 ; 2]$. During this process, some cells at the wound margin polarize and form typical membrane protrusions associated with cell migration, such as lamellipodia and filopodia. As for single cells, small GTPases within the Rho family such as Rac, Cdc42 and Rho, are highly involved in the sheet migration. Rac inhibition of the three first cellular rows adjacent to the margin has for instance been shown to completely inhibit, whereas Rho was shown to have a major impact on the regularity of the sheet migration [3]. Moreover, it is highly influenced by the surrounding cells as well as mechanical forces, but it is unclear whether it is driven by: (i) cells at the margin pulling the sheet forward, (ii) cells behind pushing the sheet forward, or (iii) a combination of these processes. Furthermore, the cells in the epithelial sheet could be assigned different roles, e.g. a pioneer or a follower role, based on the expression of cell surface receptors and cell-cell contacts. Disturbing the order of the epithelial sheet by reducing cadherin cell-cell junction has been found to decrease the orientation within the migrating monolayer and thereby diminish the follower behavior [4]. Thus, a highly sophisticated interplay based on cellular characteristics such as cell-cell junction, migration promoting protein expression, location in the monolayer and the epithelial integrity is indeed required for epithelial sheet migration.

Recently, water fluxes through membrane-anchored aquaporins (AQPs) have been proposed to play a pivotal role in cell migration (reviewed in $[5 ; 6 ; 7])$. By their local appearance and abundance they could aid cell locomotion by facilitating shape changes like protrusion formation being required for migration into narrow spaces. There are 13 different mammalian 
AQPs known and this trait appears to be applicable on most of them. In brief, the mechanism is assumed to be based on an increase in the hydrostatic pressure causing the membrane to dislocate from the cytoskeletal anchorage and thereby forcing it to protrude outward. Furthermore, dilution of the gel-like cytoplasm and G-actin monomers should create a steep concentration gradient and facilitate diffusion of new actin monomers to the polymerizing actin filaments $[5 ; 6 ; 8 ; 9]$. However, little is known about the effect of aquaporins in migration of a confluent epithelial sheet where para-cellular communication and cell-to-cell interactions via different cellular junctions are essential for monolayer tightness and epithelial integrity.

The aim of this study was to assess the effects of AQP9 on single cell and epithelial sheet migration. To achieve this, we used the canine kidney cell line MDCK-1, as a model system. Here, we stably expressed GFP-AQP9 under regulation of a CMV promoter yielding a relatively high expression. They were then subjected to single cell and epithelial sheet motility assays.

Our findings suggest that increased water fluxes promote both single cell and epithelial sheet migration. Moreover, we provide evidence for a novel mechanism of wound healing based on swelling and expansion of the monolayer. 


\section{MATERIALS AND METHODS}

\section{Preparation of stable AQP9-GFP expression in MDCK-1 cells}

Cells were cultured in Dulbeccos Modified Eagles Medium supplemented with $10 \%$ fetal bovine serum (FBS), $100 \mu \mathrm{g} / \mathrm{ml}$ streptomycin, $100 \mathrm{U} / \mathrm{ml}$ penicillin, $1 \mathrm{mM}$ Sodium Pyruvate and 2 mmol L-glutamine (all obtained from GIBCO BRL/Invitrogen Carlsbad, CA, USA). cDNA encoding for AQP9 was prepared as described in [10] and cloned into the retroviral backbone pRetroQ-AcGFP1c1 (Clontech laboratory Inc, Mountain View, CA). Retroviral particles containing GFP-AQP9 with pA10 trophism were produced in the packaging cell line GP-2 293 (Clontech) for $48 \mathrm{~h}$. The MDCK-1 cells were then incubated with the viruscontaining supernatant for $24 \mathrm{~h}$ and subsequently cultured in medium containing $4 \mu \mathrm{g} / \mathrm{ml}$ puromycin (Sigma-Aldrich, St Louise, MO). GFP-expressing cells were further sorted according to GFP fluorescence in an ARIA III cell sorter (Becton, Dickinson, Franklin Lakes, NJ). After generation of stable cell lines, they were constantly passaged simultaneously.

\section{Imaging}

For structured illumination confocal imaging and for high resolution epi-fluorescent imaging an Axiovert 200M (Zeiss, Jena, Germany) stage equipped with a structured illuminationaperture correlation unit (VivaTome, Zeiss) and 63x (NA 1.25; Zeiss) and 40x (NA 1.3; Zeiss) objectives, Axiocam Mrm CCD camera and a HXP 120 C fluorescent lamp. For laser scanning confocal imaging an Axio Observer Z1 stage was used equipped with a LSM700 (Zeiss) confocal unit and 20x (NA 0.8; Zeiss) objective. For wound healing assays, the cells were imaged on an inverted bench top JuLI stage (Saveen \& Werner, Digital Bio, Seoul, Korea) inside the incubator at $5 \% \mathrm{CO}_{2}$. To visualize single cell migration, a Nikon Ti-E staged with Perfect Focus and a 20x objective was used (Nikon, Tokyo, Japan). This system also contained a pE-2 lamp (CoolLED, Andover, UK), a micro-incubation chamber (QE-1), a 
TC-344B Heat Controller (both purchased from Warner Instruments, Boston, MA) and a Clara interlined CCD (Andor, Belfast, UK).

\section{Wound healing}

The cells (1-3 x $10^{5}$ cells $\left./ \mathrm{ml}\right)$ were seeded into the two compartments of an Ibidi Wound healing chamber (Ibidi, Martinsried, Germany). The cells were allowed to grow under regular culturing conditions for 3-5 days. After this, the insert was removed with a tweezer yielding a standardized wound of $500 \mu \mathrm{m}$. The dish was washed and subsequently imaged in serum-free medium for $10 \mathrm{~h}$. Images were acquired sequentially every 5 minutes. The images were subsequently assessed with the wound healing plug-in in the open source software Cell Profiler [11]. Individual thresholds were set to the different image sequences.

To analyze the number of cells/field of view the cells were fixed in $4 \%$ paraformaldehyde (PFA, Sigma-Aldrich) 4 days after seeding. Fixation was performed before and 1-7 h after removing the wound healing insert. They were then washed in PBS and subsequently mounted in ProLong Gold containing DAPI (Molecular probes/Invitrogen, Carlsbad, CA). Confocal images were captured at the epithelial monolayer with a 40x (NA 1.3; Zeiss) objective and the number of nuclei/field of view were counted manually in ImageJ.

\section{Actin staining}

To stain the actin cytoskeleton, the cells were fixed for $20 \mathrm{~min}$ at room temperature (RT) in 4\% PFA. They were then washed in PBS, permeabilized in 0.1\% Triton X-100 (SigmaAldrich) and washed again. Phalloidin-conjugated Alexa 568 (1:200; Molecular probes) was added to the samples that were subsequently incubated for $45 \mathrm{~min}$ at RT. Following a final wash, the cells were mounted in ProLong Gold containing DAPI.

\section{Single cell migration}

The cells were seeded in glass-bottom culture dishes (MatTek Corporation, Ashland, MA) 
starved and allowed to adhere for 10-12 h. One hour before acquisition the medium was changed to imaging medium previously described in [12] and stimulated with $50 \mathrm{nM}$ epidermal growth factor (EGF). Images were then acquired every $30 \mathrm{~s}$ for $7 \mathrm{~h}$ in both bright field and epi-fluorescence. The image sequences were subjected for analysis in the image processing software Imaris using the "Imaris track" module. In brief, a binary mask was created and the cells were subsequently tracked over time based on the center of the cell mass. Only motile single cells that were inside the field of view throughout image acquisition were subjected for analysis. The coordinates obtained from the tracking procedure were the loaded into Ibidis chemotaxis and migration tool (Ibidi) and the Euclidean and accumulated distances were calculated.

\section{Proliferation rate}

The cells were seeded at $2 \times 10^{5}$ cells $/ \mathrm{ml}$ in culture dishes and then placed on the JuLI bench top microscope inside the incubator. Image acquisition was carried out at an interval of 10 min for $100 \mathrm{~h}$. The cells were subsequently tracked over time in ImageJ and the time between two divisions was calculated.

\section{Transepithelial electrical resistance (TER)}

The cells were seeded at a density of $1-3 \times 10^{5}$ cells $/ \mathrm{ml}$ on collagen-coated Transwell filters (pore size $3 \mu \mathrm{m}$, Corning, New York, NY). The TER of the monolayer was measured 1-6 days later with a volt-ohmmeter (World Precision Instruments, Sarasota, FL).

\section{Statistical analyses}

All data is presented as mean \pm SEM. Statistical analyses are based on two tailed, nonparametric Mann-Whitney tests. The significance was rated * when $0.05>\mathrm{p}<0.01$, ** when $0.01>\mathrm{p}<0.001$ and $* * *$ when $\mathrm{p}<0.001$. 


\section{RESULTS AND DISCUSSION}

\section{Cells expressing AQP9 show increased single cell motility but migrates within smaller}

areas

The effect of AQP9 on the motility of MDCK-1 cells was assessed by generation of a stable cell line expressing GFP-AQP9. Here, AQP9 was shown to localize at the plasma membrane. Furthermore, to analyze the relation between the localization of AQP9 and actin, intensity profile plots of these molecules labeled with spectrally separated fluorophores was measured. Indeed, the intensity fluctuations between GFP-AQP9 and actin correlated while no correlation was observed between empty GFP-vector and actin (Fig. 1A). AQP9 has previously been shown to induce a filopodial phenotype [10]. These protrusions are, however usually lost during fixation (unpublished data) but were highly abundant in GFP-AQP9 expressing live cells (Fig. 1B). As a measure of cell spreading, we analyzed the area of a basal confocal section in the actin channel for both GFP-AQP9 and Mock-transfected cells. Although the mean area was fairly similar $(489 \pm 80, n=21$ and $339 \pm 30, n=15$, mean \pm SEM for AQP9 and Mock, respectively), AQP9 transfected cells ranged from 197 to $1508 \mu \mathrm{m}^{2}$ compared to 174 to $666 \mu \mathrm{m}^{2}$ for cells expressing empty GFP vector. This suggests that cells expressing AQP9, might possess enhanced ability to spread when required. Moreover, the filopodial phenotype dramatically increases the cell perimeter and thereby also the area of attachment. Indeed, AQP9 has previously been shown to augment cell adhesion [13]. 


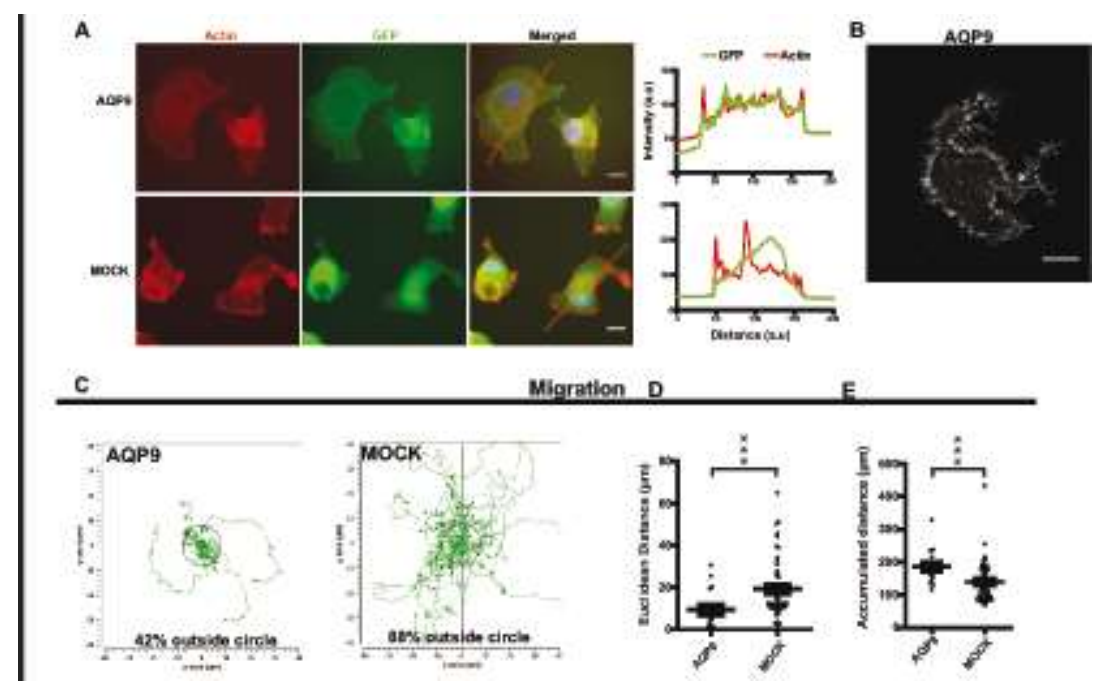

Fig. 1 Influence of AQP9 on single cell migration

(A) Images showing actin stained cells expressing GFP-AQP9 or empty GFP vector. The graphs are showing the mean intensity distribution over the line presented in the merged images. Scalebar equals $10 \mu \mathrm{m}$. (B) Confocal live cell image of a GFP-AQP9-expressing cell. (C) Tracks of migrating MDCK-1 cells expressing GFP-AQP9 or empty GFP vector after $7 \mathrm{~h}$ of migration ( $n=17$ and 52 tracks, respectively). The radius of the circle is $8 \mu \mathrm{m}$. (D) The Euclidiean distance of the tracks plotted in $\mathrm{C}(\mathrm{p}=0.0007)$. (E) The accumulated distance of the tracks plotted in $\mathrm{C}(\mathrm{p}=0.0007)$.

To stimulate single cell migration, the cells were uniformly treated with $50 \mathrm{nM}$ Epidermal Growth Factor (EGF) and imaged for $7 \mathrm{~h}$. The coordinates of the tracks were subsequently plotted and a cutoff circle with a radius of $8 \mu \mathrm{m}$ was set. The proportion of cells that migrated out of the circle was 42 and $88 \%$ for GFP-AQP9 and Mock-transfected cells, respectively (Fig. 1C, n=17 and 52, respectively). This was also clearly evident from the Euclidean distance traveled by the cells, where the end-point of cells expressing the empty GFP vector was $19.3( \pm 1.9, \mathrm{n}=52$ tracks $)$ compared to $9.4( \pm 2.2, \mathrm{n}=17$ tracks $)$ for cells expressing GFPAQP9 (Fig. 1D, p=0.0007). However, the accumulated distance during $7 \mathrm{~h}$ of migration was higher in cells with AQP9 compared to empty GFP vector (Fig. 1E, $186 \pm 12$ and 140 \pm 8 , $\mathrm{p}=0.0007)$ meaning that they were highly dynamic but indecisive concerning where to migrate. This probably reflects the highly dynamic membrane and formation of multiple protrusions that is known to be facilitated by AQP9 expression (Movie S1, [10; 13]). The 
filopodia might also enhance this effect since they are known to function as gradient sensors and therefore probably sensitizes the cells ability to detect the stimuli. The AQP9-expressing cell might thus easily detect minute differences in the uniform EGF around the cell. Since these minute differences likely changes rapidly the cell will act indecisively concerning persisted direction of migration. The GFP control cells that lack this filopodial phenotype will probably continue to migrate in the direction where they initially detected the EGF. This, however, is a subject for future studies.

\section{AQP9 facilitates wound closure}

Since expression of AQP9 was shown to facilitate the accumulated distance in single cells, we wanted to investigate the effects on epithelial sheet migration. Similar to the single cells, AQP9 was shown to localize to the plasma membrane (Fig. 2A). To visualize similarities in intensities, a cropped area of AQP9 and actin was pseudo-colored in rainbow scale; a scale that ranges from blue to red based on a low or high intensity, respectively. The fluorescence intensity fluctuations correlated between AQP9 and actin at the leading edge of cells at the wound margin (Fig. 2A insert). To study the epithelial sheet migration, a wound healing experiment was used. Here, the expression of GFP-AQP9 was shown to facilitate this process significantly (Fig 2B and C, n= 6 and 4 experiments for AQP9 and Mock, respectively, $\mathrm{p}<0.0001)$. Moreover, the motile phenotypes of AQP9 and Mock-transfected monolayer were clearly distinct; GFP-AQP9 expression resulted in a swelling monolayer pushing from the back, while the wound healing for Mock-transfected cells was more dependent on individual cell migration (Movie S2). This might indicate that the density of cells and the cell size of individual cells within the monolayer might play an important role in these instances. 
A
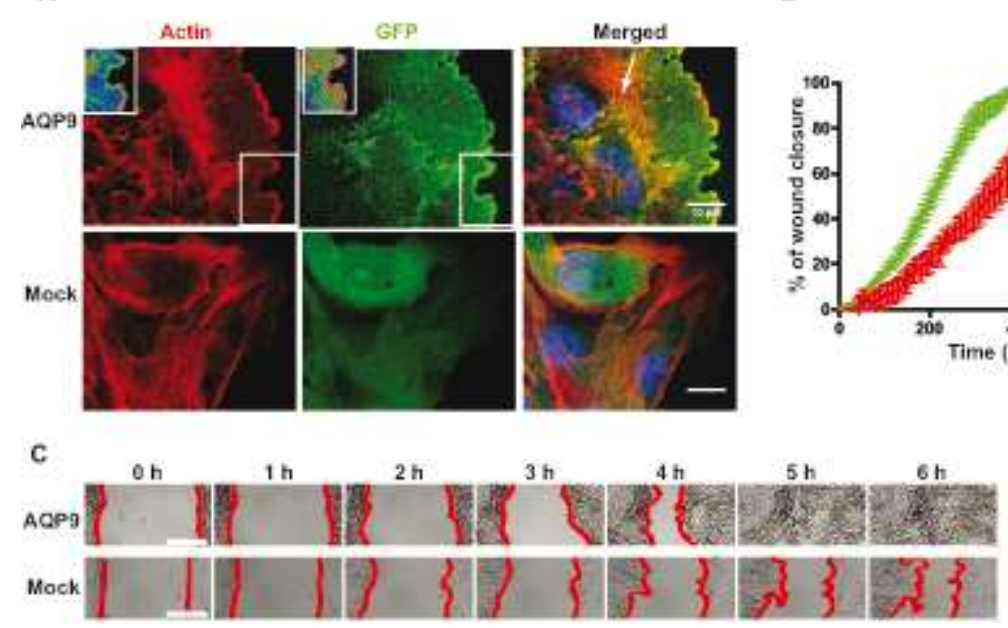

B

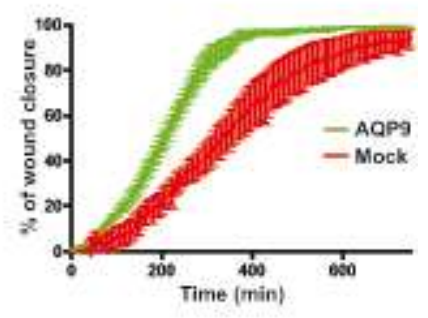

\section{Fig.2 Influence of AQP9 on wound healing}

(A) Images showing actin stained cells expressing GFP-AQP9 or empty GFP vector at the wound margin $4 \mathrm{~h}$ after removal of the wound healing insert. The insert represents a rainbow pseudo-colored area of the white box to illustrate similarities in the intensity distribution in the green and red channel. The white arrow is pointing toward a filopodia. Scale bar $10 \mu \mathrm{m}$. (B) The graph illustrates the time course of wound closure. The data is presented as mean \pm SEM, $n=6$ and 4 experiments for AQP9 and Mock-transfected cells, respectively. (C) Representative images of wound healing experiments. The red line illustrates the border of the monolayer. Scale bar equals $200 \mu \mathrm{m}$.

\section{AQP9 tightens the epithelial sheet and causes expansion of the monolayer during wound}

\section{healing}

The different motile phenotypes of the migrating epithelial sheets led us to investigate the cell size within the monolayer. Since fluxes of water across the cell membrane are known to be osmotically regulated, we hypothesized that the cell volume changes might be a mechanism underlying facilitated epithelial sheet migration. By visualizing actin at a relatively low magnification in the margin of the migrating monolayer, we observed a distinct difference upon expression of AQP9. Here, the cells were organized as a relatively uniform monolayer while the Mock-transfected cells occasionally grew in more than one layer and the sheet appeared to contain more elongated cells (Fig. 3A). This was further confirmed by projecting the apical surface of a depth-coded 3-dimensional Z-stack of a nucleus-stained monolayer, where the epithelial sheets expressing AQP9 grew in a well organized uniform monolayer in 
comparison to cells with the empty GFP vector that appeared to grow less uniform and in several layers (Fig. 3B). Thus, augmenting the ability to regulate the cell volume might improve the ability to be squeezed in a monolayer. As a measure of the cell density, we calculated the amount of nuclei/field of view in confocal images. Indeed, the undisturbed AQP-expressing monolayer contained almost twice as many nuclei/field as Mock-transfected cells (Fig. C and E, 136 \pm 8 and $75 \pm 3$ for AQP9 and Mock, respectively; $p=0.0004 ; n=9$ fields/cell type). Interestingly, in wound healing experiments, this number decreased along with the migration to $108( \pm 4)$ after $3 \mathrm{~h}(\mathrm{p}=0.0027$ versus $0 \mathrm{~h})$ and $96( \pm 7)$ nuclei/field after 7 $\mathrm{h}(\mathrm{p}=0.03$ versus $3 \mathrm{~h})$. This left the actin cytoskeleton intact suggesting that the effect was mediated through cell swelling (Fig. 3C and D). Incidentally, this was not observed in Mocktransfected cells and was not an effect of increased cell proliferation, which was $13( \pm 1)$ and $12( \pm 1)$ h between divisions for AQP9 and Mock-transfected cells, respectively (Fig. 3E; $\mathrm{p}=0.23 ; \mathrm{n}=10$ and 8 tracked cell divisions, respectively). A denser monolayer should likely increase the tightness of the epithelial sheet. We therefore analyzed the transepithelial electrical resistance across the monolayers. Here, AQP9 expression increased the resistance in comparison to control cells at three days after seeding the cells $\left(374 \pm 38 \mathrm{ohms} \mathrm{cm}^{2}\right.$ and $120 \pm 6$ ohms $\mathrm{cm}^{2}$ for AQP9 and Mock-transfected cells, respectively, $\mathrm{p}<0.0001 ; \mathrm{n}=9$ experiments). It continued to increase further until 5 days after seeding, where it reached a maximum resistance of $647( \pm 99)$ ohms $\mathrm{cm}^{2}$, while Mock-transfected cells remained nearly constant throughout the experiment (Fig. 3F). To test whether the wound healing process followed a similar pattern, we seeded AQP9 expressing cells for 1 and 3 days before initiation of the experiment. Indeed, the rate of wound closure was faster in AQP9-expressing monolayer on Day 3 in comparison to Day 1. In contrast, the wound closure of AQP9-expressing cells that were only allowed to form monolayers for $24 \mathrm{~h}$ was very similar to Mock-transfected 
monolayers that had grown for three days (Fig. 3G). These results parallel the TER-

measurements and might be explained by a denser monolayer.

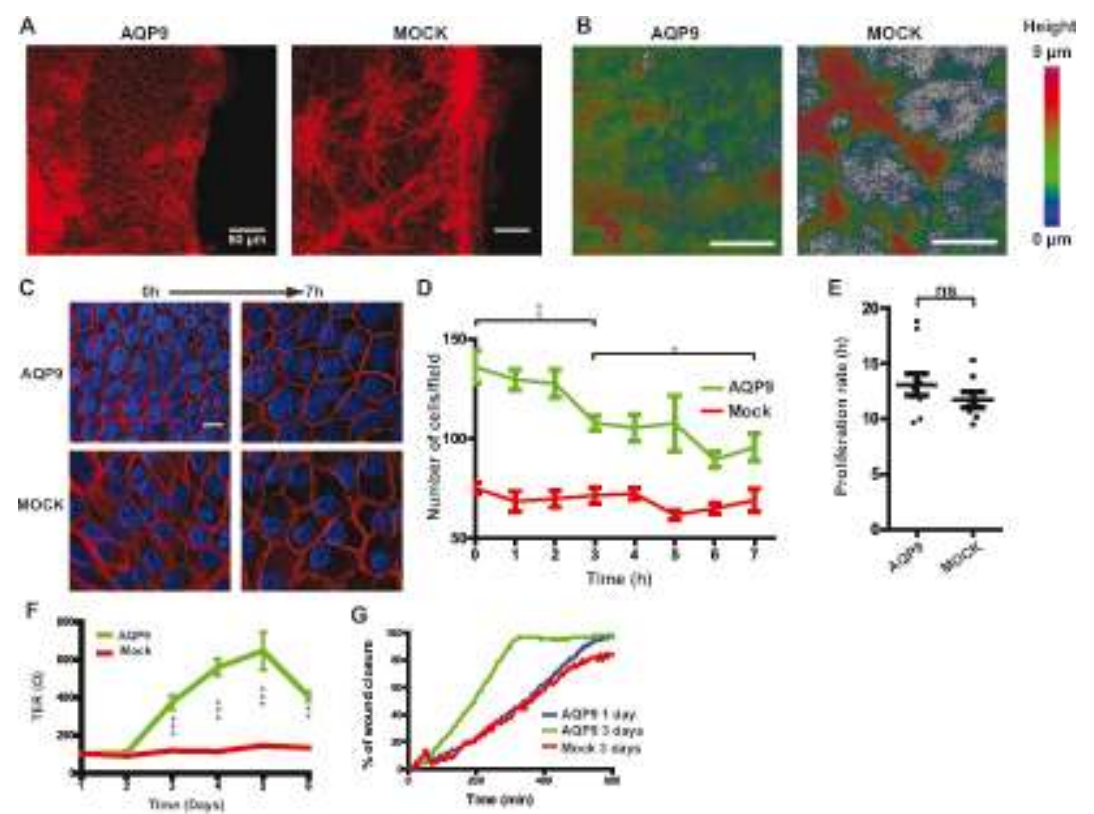

Fig. 3 Structure of the monolayer

(A) Actin-stained migrating monolayers of cells expressing GFP-AQP9 or empty GFP vector. The images are showing differences in the monolayer organization. Scale bar $50 \mu \mathrm{m}$. (B) Representative depth-coded surface projections of confocal stacks obtained from DAPIstained cells to illustrate differences in monolayer composition. (C) Representative images of actin-stained cells before, and $7 \mathrm{~h}$ after initiation of wound healing. Actin is shown in red and the nucleus is shown in blue. Scale bar equals $10 \mu \mathrm{m}$. (D) Quantification of the number of cells/field in images of fixed cells before, and 1-7 $\mathrm{h}$ after initiation of wound healing. AQP9 expressing cells displayed a significant decrease in the number of cells/field $(p=0.003$ between 0 and $3 \mathrm{~h}, \mathrm{p}=0.03$ between 3 and $7 \mathrm{~h}$ ). The data is based on more than 9 fields/cell type and time point. (E) Illustrates the proliferation rate of cells expressing GFP-AQP9 or empty GFP vector ( $\mathrm{n}=10$ and 8 tracked AQP9 and Mock-transfected cells, respectively). (F) The transepithelial electrical resistance in monolayers 1-6 days after seeding $(\mathrm{p}<0.001$ after 3 days, $n=9$ experiments). (G) Wound healing experiments of cells that were allowed to form monolayers for 1 or 3 days.

\section{Model for AQP9-augmented cell migration}

To conclude our findings, we have developed a model for AQP9-assisted cell migration at the single and multi-cellular level (Fig. 4). During individual cell locomotion, AQP9 will help induce multiple cell protrusions, viz. filopodia, lamellipodia and blebs by facilitating influx of water at these sites. This phenotype is highly motile and constantly changes shape. However, 
if an apparent chemotactic gradient is not established, the cells become indecisive concerning the direction of migration. After several proliferation cycles the cells will start to form a monolayer (Fig 4B). Fluxes of water in an out of the cells are known to efficiently regulate the cell volume. Along with proliferation within the monolayer, the water channels augments the volume regulation and hence, the ability to squeeze in a uniform monolayer accordingly. In AQP9-enriched monolayers, this results in a high cell density, which also increases the epithelial tightness and exerts an increasing force on adjacent structures, i.e. vessel wall or epithelium in the kidney or gut (Fig. 4C). After an accidental wounding of the monolayer, the epithelial integrity is temporarily disrupted, but when water is taken up by the remaining cells, the epithelial pressure will force boundary cells into the wound. Thus, a rapid swelling of the monolayer will push the sheet in the direction of the wound (Fig 4D).

In summary, our study suggests that water flux is a pivotal parameter in both single cell and multi-cellular sheet migration, and thereby plays an essential role in physiological processes during wound healing. The force created by the swelling monolayer might also be critical in events of epithelial renewal e.g. in the intestine where cells are pushed towards the lumen along with an increased division further down in the tissue.

\section{ACKNOWLEDGEMENT}

The authors are grateful to Dr. Eva Arnspang, Dr. Jonathan Brewer (University of Southern Denmark, Odense, Denmark) Bachelor student Renate Gullberg and Master student Risul Amin (Linköping University, Linköping, Sweden) for experimental help. This research was supported by the Swedish Research Council for Medicine and Health (Grant Nos 2007-3483, 2009-6649 and 2010-3045). This study was also a part of Euro-BioImaging proof-of-concept studies (Spring 2012). 


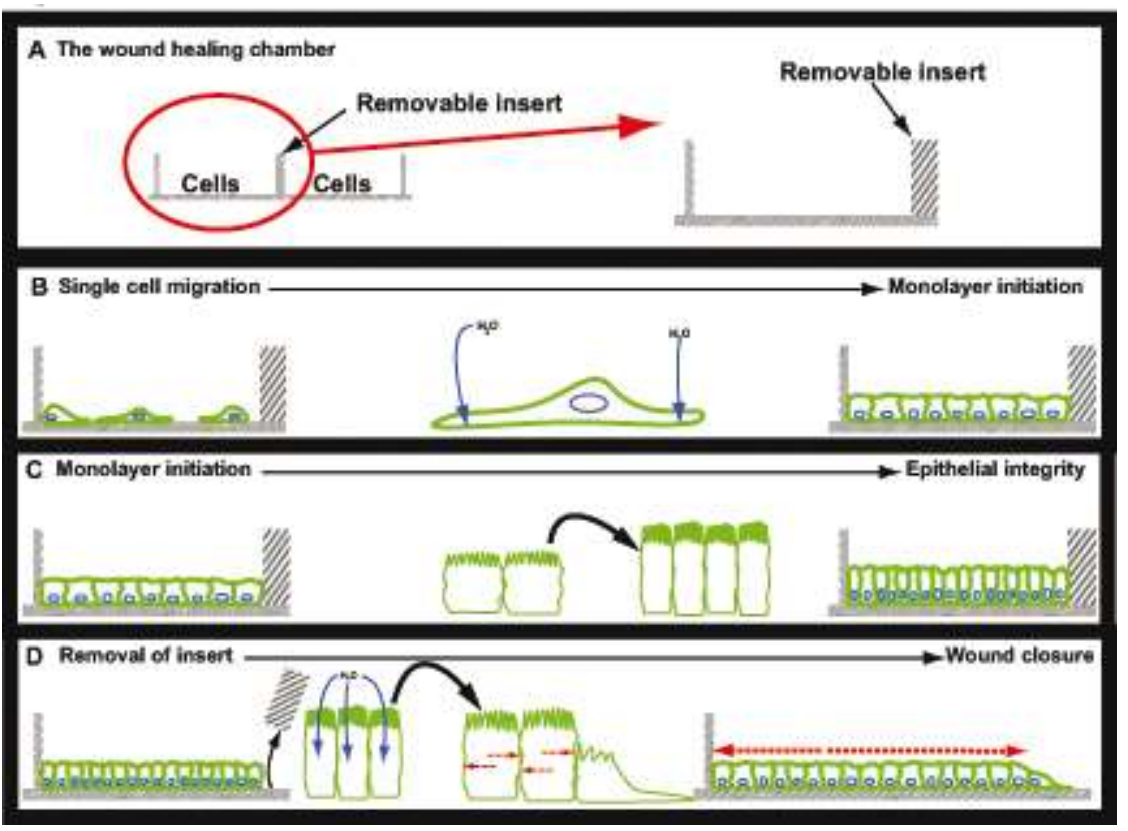

Fig. 4 Model for aquaporin (AQP9)-assisted cell migration

(A, left) Schematic illustration of the wound healing chamber. The cells are seeded into two different compartments separated by a removable insert. (A, right) A zoomed illustration of one of the compartments. (B) After seeding the cells in the wound healing chamber the cells will migrate as single cells until they proliferate or encounter another cell. This is assisted by AQP9 expression by facilitating shape changes and possibly also by sensitizing the cell to chemotactic compounds originating from adjacent cells. After several rounds of proliferation the cells will start to form a monolayer. (C) The newly formed monolayer will continue to proliferate and the cell density will increase. Along with this, the cells will also increase in height and by pumping water in an out through AQP9, they will efficiently regulate their cell volume thus enabling them to squeeze in the monolayer similarly as AQPs are assumed to facilitate shape changes in other narrow spaces. (D) Upon removal of the insert, water may rapidly flow into the cells through AQP9 and thereby induce swelling of a cell and epithelial sheet as a whole. This will create a force (indicated by red arrows) that will push the monolayer into the newly created gap and thereby facilitate rapid wound closure. 


\section{REFERENCES}

[1] J.P. Heath, Epithelial cell migration in the intestine. Cell Biol Int 20 (1996) 139-46.

[2] V. Vasioukhin, and E. Fuchs, Actin dynamics and cell-cell adhesion in epithelia. Curr Opin Cell Biol 13 (2001) 76-84.

[3] G. Fenteany, P.A. Janmey, and T.P. Stossel, Signaling pathways and cell mechanics involved in wound closure by epithelial cell sheets. Curr Biol 10 (2000) 831-8.

[4] P. Vitorino, and T. Meyer, Modular control of endothelial sheet migration. Genes Dev 22 (2008) 3268-81.

[5] M.C. Papadopoulos, S. Saadoun, and A.S. Verkman, Aquaporins and cell migration. Pflugers Arch 456 (2008) 693-700.

[6] V.M. Loitto, T. Karlsson, and K.E. Magnusson, Water flux in cell motility: expanding the mechanisms of membrane protrusion. Cell Motil Cytoskeleton 66 (2009) 237-47.

[7] A. Schwab, A. Fabian, P.J. Hanley, and C. Stock, Role of ion channels and transporters in cell migration. Physiol Rev 92 (2012) 1865-913.

[8] V.M. Loitto, T. Forslund, T. Sundqvist, K.E. Magnusson, and M. Gustafsson, Neutrophil leukocyte motility requires directed water influx. J Leukoc Biol 71 (2002) 212-22.

[9] S. Saadoun, M.C. Papadopoulos, M. Hara-Chikuma, and A.S. Verkman, Impairment of angiogenesis and cell migration by targeted aquaporin-1 gene disruption. Nature 434 (2005) 786-92.

[10] V.M. Loitto, C. Huang, Y.J. Sigal, and K. Jacobson, Filopodia are induced by aquaporin9 expression. Exp Cell Res 313 (2007) 1295-306.

[11] A.E. Carpenter, T.R. Jones, M.R. Lamprecht, C. Clarke, I.H. Kang, O. Friman, D.A. Guertin, J.H. Chang, R.A. Lindquist, J. Moffat, P. Golland, and D.M. Sabatini, CellProfiler: image analysis software for identifying and quantifying cell phenotypes. Genome Biol 7 (2006) R100.

[12] P.S. Brown, E. Wang, B. Aroeti, S.J. Chapin, K.E. Mostov, and K.W. Dunn, Definition of distinct compartments in polarized Madin-Darby canine kidney (MDCK) cells for membrane-volume sorting, polarized sorting and apical recycling. Traffic 1 (2000) 124-40.

[13] T. Karlsson, M. Glogauer, R.P. Ellen, V.M. Loitto, K.E. Magnusson, and M.A. Magalhaes, Aquaporin 9 phosphorylation mediates membrane localization and neutrophil polarization. J Leukoc Biol 90 (2011) 963-73. 